replaced MCP joint to a more extended position [2]. However, since the correlation between extension deficit of PIP join and MCP joint flexion range was not actually observed, it is unlikely that a decrease in the extension of the PIP joint is a result of compensation. There is a possibility that the procedure such as intrinsic tendon release, bone shortening by resection of metacarpal head, and centralization of extensor tendon [3], might influence the postoperative PIP joint motion. It is necessary to pay attention to changes in the ROM of the PIP joint after joint replacement arthroplasty of MCP joint.

References:

[1] Escott BG, Ronald K, Judd MG, Bogoch ER. NeuFlex and Swanson metacarpophalangeal implants for rheumatoid arthritis: prospective randomized, controlled clinical trial. J Hand Surg Am. 2010 Jan;35(1):44-51.

[2] Mannerfelt L, Andersson K. Silastic arthroplasty of the metacarpo- phalangeal joints in rheumatoid arthritis. J Bone Joint Surg 1975; 57A:484 - 489.

[3] Swanson AB. Flexible implant arthroplasty for arthritic finger joints: rationale, technique, and results of treatment. J Bone Joint Surg Am. 1972 Apr;54(3):435-55.

Acknowledgements:

Disclosure of Interest: None declared

DOI: 10.1136/annrheumdis-2017-eular.4536

\section{SAT0083 TOCILIZUMAB INDUCED CLINICAL REMISSION IN RHEUMATOID ARTHRITIS HAD MORE RESIDUAL DOPPLER SIGNALS IN COMPARISON WITH OTHER BIOLOGICS}

Y. Hara ${ }^{1}$, Y. Ishida ${ }^{1}$, Y. Yamaguchi ${ }^{1}$, Y. Yoshimine ${ }^{1}$, T. Wibowo ${ }^{1}$, Y. Manabe ${ }^{1}$,

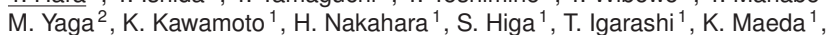
A. Ogata ${ }^{1} .{ }^{1}$ Division of Allergy, Rheumatology and Connective Tissue Diseases, Department of Internal Medicine; ${ }^{2}$ Respiratory Medicine, Department of Internal Medicine, Ntt West Osaka Hospital, Osaka, Japan

Objectives: To study the residual Power Doppler (PD) signals for assessing synovitis in CRP negative patients with rheumatoid arthritis (RA) treated with biologics.

Methods: Biologics treated RA patients who were maintained normal CRP for more than 6months (34 TNF inhibitors, 37 Tocilizumab, 23 Abatacept) were assessed by ultrasonography. Residual PD signals were assessed in MCP, PIP, and wrist joints with both hands. We assumed patients who had any PD signals in assessed joints were "residual PD signals".

Results: All treated patients with biologics maintained normal CRP for a half year $(n=94) .35 .1 \%$ of patients treated with biologics had positive PD signals. The remission rates of DAS28-ESR, CDAI, and Boolean were $68.7 \%, 62.8 \%$, and $48.9 \%$ respectively. $28.1 \%$ of patients who achieved DAS28-ESR remission had residual PD signals, $22.0 \%$ in CDAl, and $21.7 \%$ in Boolean. $23.8 \%$ of patients treated by TNF inhibitors who achieved DAS28-ESR remission had residual PD signals, $34.6 \%$ in Tocilizumab, and $20.0 \%$ in Abatacept. In case of setting the DAS28-ESR remission less than $3.0,30.2 \%$ of patients who achieved DAS28-ESR remission had residual PD signals, $29.0 \%$ in less than 2.0 , and $12.5 \%$ in less than 1.5. The patients who have no tender or swollen joints and the excellent patient oriented global health assessment (VAS score was zero) had fewer residual PD signals $(23.4 \%$ vs $58.6 \%$ in tender joints, $26.7 \%$ vs $66.7 \%$ in swollen joints, and $13.3 \%$ vs $44.4 \%$ in patient VAS).

Table 1. Rate of remission and residual PD signals

\begin{tabular}{lcccc}
\hline & All biologics & TNF inhibitors* & Tocilizumab & Abatacept \\
\hline Patients number & 94 & 34 & 37 & 23 \\
Residual PD signals** & $33 / 94(35.1 \%)$ & $9 / 34(26.4 \%)$ & $15 / 37(40.5 \%)$ & $9 / 23(39.1 \%)$ \\
DAS28-CRP remission & & & & \\
$\quad$ Achieved*** & $78 / 94(83.0 \%)$ & $32 / 34(94.1 \%)$ & $31 / 37(83.8 \%)$ & $15 / 23(65.2 \%)$ \\
$\quad$ Residual PD signals & $22 / 78(28.2 \%)$ & $8 / 32(25.0 \%)$ & $10 / 31(32.3 \%)$ & $4 / 15(26.7 \%)$ \\
DAS28-ESR remission & & & & \\
$\quad$ Achieved & $57 / 94(68.7 \%)$ & $21 / 34(72.4 \%)$ & $26 / 37(78.8 \%)$ & $10 / 23(47.6 \%)$ \\
$\quad$ Residual PD signals & $16 / 57(28.1 \%)$ & $5 / 21(23.8 \%)$ & $9 / 26(34.6 \%)$ & $2 / 10(20.0 \%)$ \\
CDAl remission & & & & \\
$\quad$ Achieved & $59 / 94(62.8 \%)$ & $26 / 34(76.5 \%)$ & $23 / 37(62.2 \%)$ & $10 / 23(43.5 \%)$ \\
$\quad$ Residual PD signals & $13 / 59(22.0 \%)$ & $5 / 26(19.2 \%)$ & $7 / 23(30.4 \%)$ & $1 / 10(10.0 \%)$ \\
Boolean remission & & & & \\
$\quad$ Achieved & $46 / 94(48.9 \%)$ & $21 / 34(61.8 \%)$ & $17 / 37(45.9 \%)$ & $8 / 23(34.8 \%)$ \\
$\quad$ Residual PD signals & $10 / 46(21.7 \%)$ & $5 / 21(23.8 \%)$ & $4 / 17(23.5 \%)$ & $1 / 8(12.5 \%)$ \\
\hline
\end{tabular}

${ }^{*}$ TNF inhibitors (Infliximab $(n=6)$, Etanercept $(n=14)$, Adalimumab $(n=7)$, Golimumab $(n=4)$, Certolizumab pegol $(n=3)) .{ }^{* \star}$ Residual PD signals: patients who had any PD signals in asessed joints. ${ }^{\star \star \star}$ Achieved: remission rate in biologics treated patients who were maintained normal CRP for a half year.

Conclusions: The patients who achieved clinical remission had residual PD signals. Patients with Tocilizumab induced remission had more residual PD signals compared with TNF inhibitor or Abatacept induced remission. More strict criteria of clinical remission may reduce residual PD signals in patients treated by Tocilizumab.

Disclosure of Interest: None declared

DOI: 10.1136/annrheumdis-2017-eular.2417

\section{SAT0084 CHARACTERISTICS OF PATIENTS WHO RESPOND POORLY TO REDUCTION OF BIOLOGICAL DISEASE-MODIFYING ANTI-RHEUMATIC DRUGS IN RHEUMATOID ARTHRITIS; RT-4 STUDY POST HOC ANALYSIS}

Y. Urata ${ }^{1}$, S. Abe ${ }^{2}$, B. Devers ${ }^{2}$, Y. Nakamura ${ }^{3}$, H. Takemoto ${ }^{4}$, K.-I. Furukawa ${ }^{5}$ ${ }_{1}^{1}$ Department of Rheumatology, Tsugaru General Hospital, Gosyogawara; ${ }^{2}$ Marketing Department, Diagnostics Division, Sekisui Medical Co., Ltd., Tokyo; ${ }^{3}$ Department of Orthopedic Surgery; ${ }^{4}$ Department of Dermatology, Tsugaru General Hospital, Gosyogawara; ${ }^{5}$ Department of Pharmacology, Hirosaki University Graduate School of Medicine, Hirosaki, Japan

Background: A number of studies have shown that reduction of biological disease-modifying anti-rheumatic drugs (bDMARDs) is possible for rheumatoid arthritis (RA) patients in whom bDMARD treatment has induced clinical remission or low disease activity. However, there have been few studies which have evaluated the background characteristics of patients who have difficulty in reducing bDMARDs despite being in clinical remission or having low disease activity.

Objectives: To clarify the characteristics of RA patients who have difficulty with bDMARD reduction despite maintaining clinical remission in the rT-4 study.

Methods: This study is a post-hoc analysis of the rT-4 study. Briefly introducing the rT-4 study: 209 RA patients demonstrating both SDAI remission and MMP-3 normalization using bDMARDs for $\geq 3$ months were randomly allocated to one of four strategy groups: Standard care (SC group; $n=50$ ); SDAl-driven therapy $(n=53)$; MMP-3-driven therapy $(n=55)$; or both SDAl and MMP-3-driven therapy group (Twin; T group; $n=51$ ). Dose reduction methodology (every 3 months): ettanercept (ETN) - period between injections increased by one week; tocilizumab (TCZ) - dose reduced by $80 \mathrm{mg}$ or period between injections increased by one week; up to a minimum dose of: ETN - 25 mg every 5 weeks; TCZ - $80 \mathrm{mg}$ every 5 weeks or $162 \mathrm{mg}$ every 5 weeks. The dose was reverted to the previous level in the event that the target scores were exceeded, and the lower dose was eventually reattempted after the target was re-achieved. The primary outcome was the difference in the proportion of patients who maintained remission at 12 months among the four groups, compared against a non-inferiority margin of $10 \%$. The results of the rT- 4 study revealed that a twin target strategy can achieve effects non-inferior to standard care with regard to maintaining clinical remission. Specifically, subgroup analysis was carried out between the T and SC groups of the rT-4 study. A factor was defined as significant when the difference in the proportion of patients who maintained remission at 12 months in the two groups across subgroups, exceeded $10 \%$.

Results: The requisite margin was recognized only for rheumatoid factor (RF) negativity (35.2\%; $95 \%$ Cls of 14.4 to 65.0$)$. Others factor were not identified. Disease activity is likely to be affected by even a small dose reduction of bDMRADS in RA patients who are RF negative, regardless of the presence or absence of anti-citrulline antibody, and it can probably be explained as a nocebo effect. Nocebo effects occur in studies which investigate the effectiveness of drug reduction, and we believe that the nocebo effect is prone to happen in RF-negative patients.

Conclusions: The twin target strategy can be used to reduce the dose of bDMARDs for RA patients while maintaining the effectiveness of said bDMARDs, with the exception of RF negative patients.

Disclosure of Interest: None declared

DOI: 10.1136/annrheumdis-2017-eular.2498

\section{SAT0085 A STUDY ON CHARACTERISTICS OF RHEUMATOID ARTHRITIS PATIENTS ACHIEVING DEPRESSION REMISSION WITH 6 MONTHS OF BIOLOGIC AGENT TREATMENT}

Y. Miwa ${ }^{1}$, N. Yajima ${ }^{1}$, T. Isozaki ${ }^{1}$, R. Takahashi ${ }^{1}$, Y. Miura ${ }^{1}$, Y. Ikari ${ }^{1}$ M. Hatano ${ }^{1}$, T. Hayashi ${ }^{1}$, T. Kasama ${ }^{1}$, K. Sanada ${ }^{2}$ on behalf of ASHURA Registry Group. ${ }^{1}$ Division of Rheumatology, Department of Medicine; ${ }^{2}$ Department of Psychiatry, Showa University School of Medicine, Tokyo, Japan

Background: Approximately $15 \%$ of RA patients also suffer from depression, with an odds ratio of $1.42(95 \% \mathrm{Cl} 1.3-1.5)$ compared with healthy people. A previous study reported that biological agents can improve the depressed state associated with RA. Although previous studies have been cross-sectional, there were no reports that analyzed factors that led to depression remission.

Objectives: To examine the relationship between baseline factors and depression remission after six-month biologic agent treatment in rheumatoid arthritis (RA) patients.

Methods: The subjects were 384 RA patients treated with biologic agents. The following patient's characteristics were investigated: age, gender, number of previous drugs, disease duration, type of biologic agents, baseline steroid dosage, methotrexate dosage and serum matrix metalloproteinase-3 (MMP-3) levels. For evaluation, we used the Simplified Disease Activity Index for RA disease activity; the Health Assessment Questionnaire Disability Index (HAQ-DI) score for activities of daily living; the Short Form-36 for nonspecific health-related quality of life; and Hamilton Depression Rating Scale (HAM-D) scores for depression status. Depression remission was defined by HAM-D $\leqq 7$ after 6 months of treatment. The subjects were divided into two groups according to the presence or absence of depression, and a retrospective study was performed.

Results: We included 152 patients in the analysis. Two hundred thirty-two 